\title{
Preparation and Viscoelastic Properties of Composite Fibres Containing Cellulose Nanofibrils: Formation of a Coherent Fibrillar Network
}

\author{
Tobias Moberg, ${ }^{1,2,3}$ Hu Tang, ${ }^{4}$ Qi Zhou, ${ }^{2,3,4}$ and Mikael Rigdahl ${ }^{1,2,3}$ \\ ${ }^{1}$ Department of Materials and Manufacturing Technology, Chalmers University of Technology, 41296 Gothenburg, Sweden \\ ${ }^{2}$ Wallenberg Wood Science Center, Chalmers University of Technology, 41296 Gothenburg, Sweden \\ ${ }^{3}$ Royal Institute of Technology, 10044 Stockholm, Sweden \\ ${ }^{4}$ Royal Institute of Technology, School of Biotechnology, 10044 Stockholm, Sweden
}

Correspondence should be addressed to Mikael Rigdahl; mikael.rigdahl@chalmers.se

Received 15 March 2016; Revised 2 June 2016; Accepted 16 June 2016

Academic Editor: Ilker S. Bayer

Copyright (C) 2016 Tobias Moberg et al. This is an open access article distributed under the Creative Commons Attribution License, which permits unrestricted use, distribution, and reproduction in any medium, provided the original work is properly cited.

\begin{abstract}
Composite fibres with a matrix of poly(ethylene glycol) (PEG) and cellulose nanofibrils (CNF) as reinforcing elements were produced using a capillary viscometer. Two types of CNF were employed: one based on carboxymethylated pulp fibres and the other on TEMPO-oxidized pulp. Part of the latter nanofibrils was also grafted with PEG in order to improve the compatibility between the CNF and the PEG matrix. The nominal CNF-content was kept at 10 or 30 weight-\%. The composite fibres were characterized by optical and scanning electron microscopy in addition to dynamic mechanical thermal analysis (DMTA). Evaluation of the storage modulus indicated a clear reinforcing effect of the CNF, more pronounced in the case of the grafted CNF and depending on the amount of CNF. An interesting feature observed during the DMTA-measurements was that the fibrils within the composite fibres appeared to form a rather coherent and load-bearing network which was evident even after removing of the PEG-phase (by melting). An analysis of the modulus of the composite fibres using a rather simple model indicated that the CNF were more efficient as reinforcing elements at lower concentrations which may be associated with a more pronounced aggregation as the volume fraction of CNF increased.
\end{abstract}

\section{Introduction}

The interest in using polymers and composites based on renewable resources has increased tremendously during recent years; compare, for example, [1]. This is coupled to the aim towards a more sustainable society and decoupling of the dependence of fossil-based raw materials. The development and use of poly(lactic acid), which can be derived from starch or sugar, can be used as an example here, as well as thermoplastic starch; see, for example, [2,3]. Natural fibres as reinforcing elements in composite materials also contribute to the aim towards more sustainable materials. An obvious example of such elements is cellulosic fibres, such as wood and plant fibres. The idea of using cellulosic materials in different forms as reinforcements or fillers is certainly not new [4], but modifications and refinements of the material have during recent years opened up new possibilities with regard to different applications.

Cellulose fibres can be said to consist of a number of nanofibrils or microfibrils and fibril aggregates which are important building blocks for the fibres. These fibrils can be separated from the cellulose fibres by aqueous homogenization process at high pressure/high shear rates, often combined with a pretreatment of the fibres using, for example, enzymes [5], 2,2,6,6-tetramethylpiperidine-1-oxyl (TEMPO) radicalmediated oxidation [6], or carboxymethylation [7] in order to reduce the energy consumption. The fibrils are denoted as cellulose nanofibrils (CNF) or nanofibrillated cellulose (NFC), and the term microfibrillated cellulose (MFC) is sometimes also used. CNF have been suggested for several different applications and products, for example, hightoughness paper [8-11], barrier material (lowering the oxygen 
and oil permeability) [12-14], aero- and hydrogels [15-18], and reinforcing elements in composite materials $[15,19-24]$. In this context it is certainly of interest that, as pointed out by Lee et al. [19], the number of publications on cellulose nanocomposites has increased exponentially over the recent years, but already in the 1980s initial attempts were made to use nanocellulose as a reinforcing element in thermoplastics; see, for example, [24]. Several types of matrix polymers have been combined with CNF (and similar elements), such as poly(lactic acid), poly(vinyl alcohol), epoxy, starch, polyurethane, and unsaturated polyester, just to mention a few.

After the homogenization process, the concentration of the CNF is usually quite low, of the order of 2-5 weight-\% (wt-\%) or lower, in order to minimize or avoid aggregation. The aggregation is associated with colloidal interactions and the entanglement of the fibrils. Drying of the suspensions can lead to hornification of the material and redispersing the dry fibril aggregates is very difficult. This behaviour prevents a straightforward incorporation of CNF into commodity thermoplastic matrices, which in most cases are hydrophobic. This consequently makes manufacturing of nanocomposites using conventional processing techniques such as extrusion and injection moulding challenging [25].

Closely connected to the processability of materials are their rheological properties. Even at low concentrations, the CNF suspensions exhibit quite a complex rheological behaviour; they are shear-thinning, at least at not too high shear rates, and elastic in nature; compare, for example, $[5,26-28]$. Both the shear viscosity and the viscoelastic parameters of the CNF suspensions are affected by additives like salts, carboxymethyl cellulose, cationic starch, and polymethacrylates $[29,30]$. The CNF suspensions, with a fibril content exceeding the percolation threshold, exhibit a gel-like behaviour with a yield stress, a storage modulus greater than the loss modulus, and the moduli being rather insensitive to changes in the measuring frequency, for example, $[5,26]$, and to moderate changes in temperature, up to $80^{\circ} \mathrm{C}[5,31]$. When subjected to an extensional flow, the CNF suspensions exhibited quite a high extensional viscosity with a Trouton ratio which significantly exceeded three [32].

The increased interest in CNF (cf. [15]) is to some extent coupled to their potentially good mechanical performance. Their tensile modulus (including values of the cellulose crystal) has been estimated to be in the range 100 to $160 \mathrm{GPa}$ $[19,33]$ obviously making them interesting as reinforcing elements in polymer matrices. For comparison, typical values of the modulus in the longitudinal direction for glass fibres and aramid fibres are 70 and $125 \mathrm{GPa}$, respectively [34].

The tensile modulus of nanopapers and wet-spun-fibres produced from CNF or similar elements has been reported to be between about 15 and somewhat higher than $30 \mathrm{GPa}$ $[10,35-37]$. In order to utilize the potentially high modulus of the $\mathrm{CNF}$ in a polymer composite, the reinforcing elements should be well dispersed and the adhesion between the matrix and the elements should be sufficient [34]. Aggregation of the CNF leads to lower specific area, a less efficient stress transfer between the matrix and the fibrils, and thus a less efficient use of modulus of fibrils; compare [19]. In the case of unsaturated polyester containing CNF, Ansari and coworkers [23] evaluated an effective tensile modulus of the CNF and found it to decrease from $42 \mathrm{GPa}$ at a CNF-content of $16 \mathrm{wt}-\%$ down to $25 \mathrm{GPa}$ at $45 \mathrm{wt}-\% \mathrm{CNF}$. It was speculated that aggregation of the CNF was associated with the decrease in modulus with increasing amounts of CNF, thus deceasing the efficiency of the CNF. Ansari et al. [38] also noted a similar behaviour with regard to the effective modulus in the case of CNFcontaining epoxy. In a recent work, Tang et al. [39] prepared oriented ribbon-shaped composites consisting of CNF with grafted poly(ethylene glycol). The effective modulus of the $\mathrm{CNF}$ was in that case estimated to be of the order of $55 \mathrm{GPa}$ at a volume fraction CNF of 0.585 in the polymer composite.

The aim of the present study is to evaluate the possibility of producing composite fibrous materials containing CNF using a rather simple technique based on capillary viscometry. In principle, the technique used for the manufacturing can, after some modifications, be scaled up in a straightforward manner in order to produce significant amounts of these composite macrofibres. The viscoelastic properties of the composite fibres in the solid state and the reinforcing efficiency of the CNF were evaluated using dynamic-mechanical thermal analysis (DMTA). The polymer used for the matrix material was poly (ethylene glycol) (PEG). The polymer is dissolved in the aqueous CNF suspension in order to retard the aggregation (and collapse) of the nanofibrils during drying of the system, thus contributing to the homogeneity of the composite and enhancing the efficiency of the CNF with regard to the stiffness of the final composite. Two kinds of CNF were employed and in a series of experiments also CNF grafted with PEG, according to the procedure outlined in [39], were used. The grafted PEG should improve the compatibility with the PEG used for the matrix thus providing a more stable interphase region. In addition to the stiffness of the final composite as determined using DMTA, the rheological behaviour of the CNF-polymer suspensions was assessed since it reflects possible interactions between the fibrils and the polymer as well as providing useful information regarding the processability of the composite system. The integrity of the formed network of CNF in the composite fibres was assessed through the removal of the PEG-phase. In principle, composite fibres of the kind considered here can be used as reinforcing elements in other types of composite materials.

\section{Materials}

Two different types of nanofibrillated (or microfibrillated) cellulose $(\mathrm{CNF})$ were used in this work. One was prepared by high pressure homogenization of aqueous wood fibre suspensions (softwood sulphite dissolving pulp from Domsjö Fabriker, Sweden) and kindly supplied by Innventia $\mathrm{AB}$, Sweden. The pulp was carboxymethylated before being passed through a high pressure homogenizer. Due to the carboxymethylation, the resulting fibrils with a width in the range 5-10 nm were negatively surface charged, corresponding to a degree of substitution close to 0.1 . The preparation and the properties of the CNF are described in more detail by Wågberg et al. [7] and this type was denoted as CCNF. The concentration of the cellulose in the as-received 
suspension was 2.2 weight percent (wt-\%). The other suspension, denoted as T-CNF, was prepared by TEMPO-mediated oxidation of the pulp fibres (softwood sulphite pulp provided by Nordic Paper); a part of this suspension was grafted with poly(ethylene glycol) onto the pulp fibres as described in [39]. The grafting was achieved through carbodiimidemediated amidation with amino-terminated poly(ethylene glycol). Aqueous suspensions of the chemically modified fibres were mechanically disintegrated in order to obtain suspensions of nanofibrillated cellulose with a width of the order of $4 \mathrm{~nm}$ for the nongrafted fibrils. The TEMPOoxidized fibres had total carboxylate content of $1.5 \mathrm{mmol} / \mathrm{g}$ cellulose, corresponding to surface degree of substitution of 0.5 [39]. The amount of grafted poly(ethylene glycol) was $33 \mathrm{wt}-\%$. In the suspension, this was found to correspond to a width of about $5 \mathrm{~nm}$ in the case of the grafted fibrils [39]. The CNF concentration of these suspensions (from the TEMPOoxidized pulp fibres) was 0.2 wt- $\%$.

The polymer used as a matrix in the composite material was poly(ethylene glycol), PEG, or poly(ethylene oxide), PEO, from Clariant International Ltd, Muttenz, Switzerland, grade $35000 \mathrm{~S}$. This polymer is water-soluble enabling a rather straightforward mixing into the CNF suspension and providing a better compatibility with cellulose than commodity thermoplastics which normally are quite hydrophobic. The PEG-grade used in this work had, according to the supplier, an average molecular weight of $35000 \mathrm{~g} / \mathrm{mole}$, a density of $1.20 \mathrm{~g} / \mathrm{cm}^{3}$, and a melting point of at least $57^{\circ} \mathrm{C}$.

2.1. Preparation of Composite Fibres. For producing the composite material, a certain amount (depending on the aimed cellulose content) of PEG was dissolved in the CNF suspension. The polymer-containing suspension was then dried at $65^{\circ} \mathrm{C}$ until the weight of the material was the same as the calculated dry weight of the sample. This composite was then used for producing the composite fibres. Two nominal amounts of C-CNF in the PEG matrix were used here: 10 and $30 \mathrm{wt}-\%$ (dry state). In the case of the TEMPO-oxidized CNF grafted with PEG, only $30 \mathrm{wt}-\%$ was added; this means that with the grafted fibrils the cellulose content was only $20 \mathrm{wt}-$ $\%$ since one-third of those fibres consisted of grafted PEG; compare [39].

The composite fibres were produced using a capillary viscometer (Göttfert Rheograph 2002, Germany). The use of a capillary viscometer allowed for an estimation of the viscosity of the composite compound as the fibres were formed. The capillary used had a diameter of $2 \mathrm{~mm}$, a length of $20 \mathrm{~mm}$, and an entrance angle of $90^{\circ}$. Smaller diameters of the capillary led to an excessive pressure build-up in the barrel. The temperature was set to $63^{\circ} \mathrm{C}$. Lower temperatures resulted in an incomplete melting of the polymer and when increasing the temperature above $66-67^{\circ} \mathrm{C}$, the viscosity of the melt became too low to allow for a proper fibre formation.

Seven different speeds of the piston pushing the material through the capillary were used, from 0.01 to $1 \mathrm{~mm} / \mathrm{s}$ corresponding to shear rates between 1.44 and $144 \mathrm{~s}^{-1}$ in case of the used capillary. The pressure was measured by a pressure transducer in the barrel close to the capillary. The pressure readings and the corresponding piston speeds were used to calculate the apparent viscosity in the usual manner. The piston speed was increased when the pressure reading had stabilized. The fibres obtained at the highest piston speed were used for the dynamic-mechanical testing; see below. PEGfibres without CNF, to be used as a reference, were produced in a corresponding manner.

2.2. Dynamic-Mechanical Thermal Analysis (DMTA). The viscoelastic properties, given by the storage and loss moduli in tension, of the fibres from the capillary viscometer were determined by dynamic-mechanical thermal analysis (DMTA) at an applied frequency of $1 \mathrm{~Hz}$ using a Rheometrics RSA-2 equipment. The specimens were subjected to two different types of measurements: a strain sweep and a thermal sweep. The strain sweep was performed at room temperature and ambient pressure and the storage $\left(G^{\prime}\right)$ and loss moduli $\left(G^{\prime \prime}\right)$ as well as the mechanical loss factor $(\tan \delta)$ were determined as functions of an increasing applied strain amplitude. At low strain amplitudes, the moduli were independent of the deformation, but when a "critical" strain amplitude was exceeded, they decreased with increasing strain, as observed earlier [26, 27]. Below the critical value, the fibres thus exhibited a linear viscoelastic behaviour. For each sample, the strain sweep was repeated three times (using three different specimens) and the variation in storage modulus between the specimens was less than 15\% (standard deviation). The diameter of the fibre was measured using a digital calliper at three different positions along its length and the variation was typically $1 \%$.

In the temperature sweep, the maximum applied strain amplitude was kept within the linear viscoelastic region (given by the strain sweep). The starting temperature was $25^{\circ} \mathrm{C}$ (room temperature) and the viscoelastic parameters were determined as a function of temperature at a heating rate of $2^{\circ} \mathrm{C} / \mathrm{min}$ up to $120^{\circ} \mathrm{C}$. For each sample, the temperature sweep was in most cases repeated three times (using different specimens). The variation in storage modulus between the specimens was $20 \%$ or less (standard deviation).

2.3. Scanning Electron Microscopy (SEM). The cross sections of fractured fibres as well as their surface structure were visually examined in a scanning electron microscope (SEM). The samples were fractured after being submerged in liquid nitrogen and the fracture surfaces were then coated with an approximately $5 \mathrm{~nm}$ thick gold layer using a Sputter Coater S150B, BOC Edwards, UK. The SEM used in this work was a digital scanning electron microscope, Carl Zeiss DSM 940, Germany.

2.4. The CNF-Content in the Extrudates. In order to estimate the actual amount of CNF in the composite fibres, some part of the fibres nominally containing $30 \mathrm{wt}-\% \mathrm{C}$-CNF was immersed in an excess amount of deionized water for at least 24 hours. This was done in order to dissolve the PEG. The suspension was then filtered through a filter paper and both the solid residue and the filtrate were collected. The solid residue was again dissolved in deionized water for at least 24 hours and once again filtered and collected. This solid residue was then dried at low pressure and $65^{\circ} \mathrm{C}$ until its weight was 


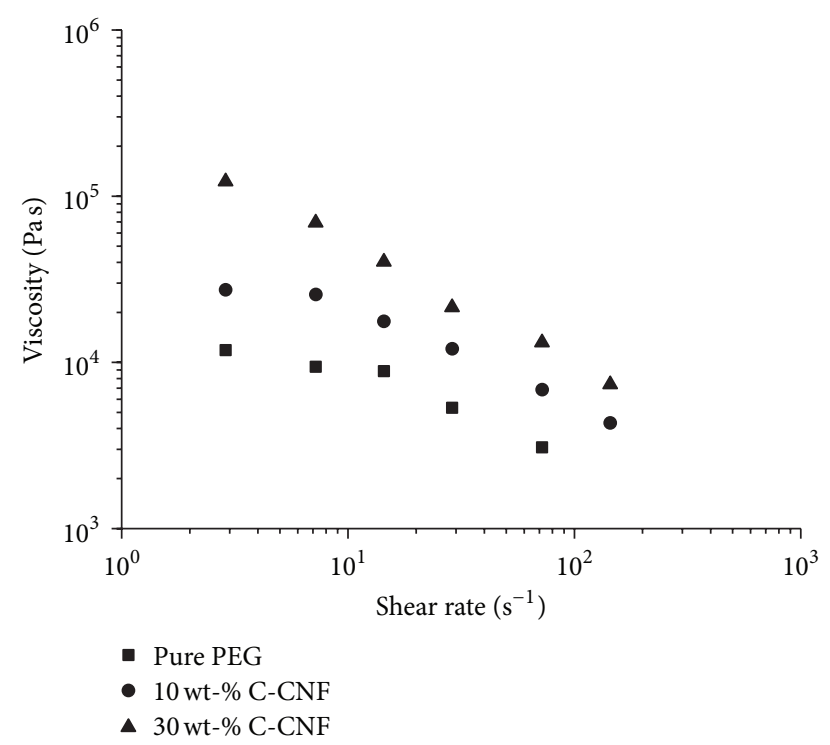

FIgURE 1: The shear viscosity as a function of shear rate at $63^{\circ} \mathrm{C}$ for unfilled and C-CNF-containing PEG.

constant. The weight of the residue corresponded to $28 \mathrm{wt}-\%$ of the initial weight of the sample which is quite close to the nominal weight fraction of the CNF in the fibres. Thus it may be reasonable to conclude that the formation of the composite fibres in the capillary viscometer did not significantly change the CNF-content.

\section{Results}

3.1. Melt Viscosity of the Composite Melts. Figure 1 shows the viscosity as a function of the shear rate at $63^{\circ} \mathrm{C}$ for unfilled PEG and for the composite melts containing nominally 10 and $30 \mathrm{wt}$ \% carboxymethylated CNF (denoted as C-CNF). As expected, the viscosity at a given shear rate increased with increasing C-CNF concentration; compare, for example, $[40,41]$. Except for the melt containing the highest amount of CNF, there was a tendency for the melts to approach a Newtonian plateau at low shear rates. At higher shear rates, all specimens exhibited a shear-thinning behaviour, which is not unexpected.

With exception of the low shear rate region, the results shown in Figure 1 can be fitted to a power-law type of relation; that is,

$$
\eta(\dot{\gamma})=K(\dot{\gamma})^{n-1},
$$

where $\eta$ is the shear viscosity, $\dot{\gamma}$ the shear rate, $K$ the consistency, and $n$ a flow index. The latter two are material parameters. Table 1 gives $K$ and $n$ for the different materials used here and it is obvious that the degree of shear-thinning increased as the amount of C-CNF increased, since the flow index decreased. This might be due to a more pronounced orientation and disentanglement of the fibrils at higher concentrations as the shear rate increases. A similar behaviour can be noted for other polymeric systems containing cellulose fibres; compare [40].
TABLE 1: The power-law parameters for PEG-melts containing CNF at $63^{\circ} \mathrm{C}$.

\begin{tabular}{lcc}
\hline Material & Consistency $K, \mathrm{kPa} \mathrm{s}^{n}$ & Flow index $n$ \\
\hline Unfilled PEG & 21.2 & 0.58 \\
10 wt-\% C-CNF & 85.4 & 0.41 \\
30 wt-\% C-CNF & 270.6 & 0.28 \\
30 wt-\% nongrafted T-CNF & 439.6 & 0.31 \\
30 wt-\% grafted T-CNF & 124.3 & 0.44 \\
\hline
\end{tabular}

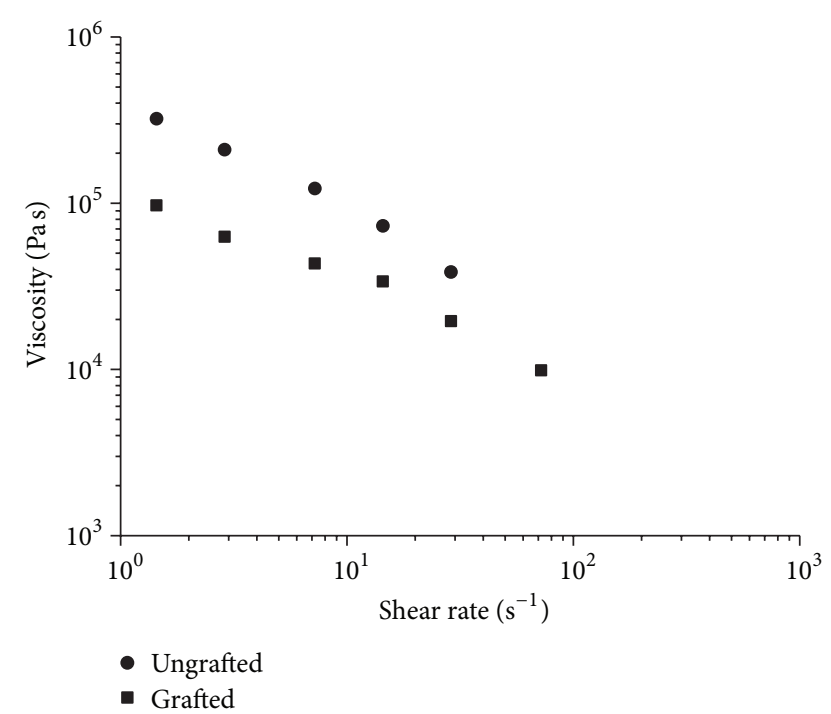

FIGURE 2: The shear viscosity as a function of shear rate at $63^{\circ} \mathrm{C}$ for the composite melts containing TEMPO-oxidized CNF, with and without grafted PEG. The nominal CNF-content was $30 \mathrm{wt}-\%$.

The viscosity of the composite melt containing $30 \mathrm{wt}-\%$ TEMPO-oxidized CNF (denoted as T-CNF) was also measured during the fibre formation in the capillary viscometer. The viscosity of these melts (with nongrafted as well as grafted $\mathrm{CNF}$ ) is shown as a function of the shear rate at $63^{\circ} \mathrm{C}$ in Figure 2; see also Table 1.

The viscosities of the melts containing $30 \mathrm{wt}-\% \mathrm{C}-\mathrm{CNF}$ and nongrafted T-CNF were not too different, although the viscosity of the latter was somewhat higher at a given shear rate. Whether the flexible and slightly more slender T-CNF gave a more flocculated structure thus enhancing the viscosity may be speculated, since it is known that aggregation normally will lead to an increase in viscosity [41]. The different surface charge character between C-CNF and T-CNF may also play a role here; compare [41]. Grafting PEG on the TCNF reduced the viscosity significantly and also diminished the shear-thinning character of the composite melt. An improved compatibility between the grafted fibres and the polymer matrix, leading to less agglomerated fibril structure, may be one reason here $[41,42]$, but reduction of the cellulose content from 30 to $20 \mathrm{wt}$ \% can also provide an explanation, at least partially.

3.2. Microscopy Studies of the Composite Fibre Structure. Figure 3 is a representative collection of optical micrographs 


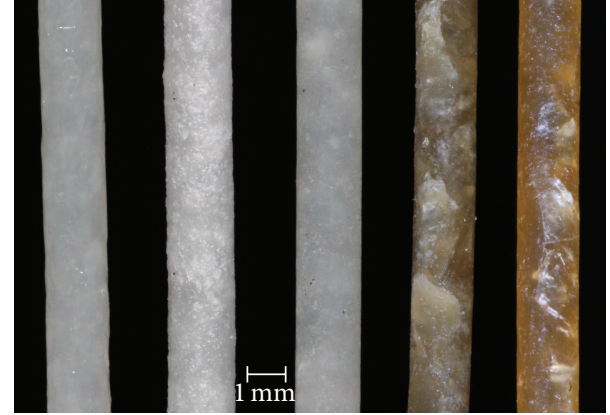

Figure 3: Optical micrographs of the extruded composite fibres. From left to right: unfilled PEG, fibre with $10 \mathrm{wt}-\% \mathrm{C}-\mathrm{CNF}$, fibre with 30 wt- $\%$ C-CNF, fibre with 30 wt- $\%$ nongrafted T-CNF, and fibre with $30 \mathrm{wt}-\%$ grafted T-CNF. The diameter of the fibres was $2 \pm 0.02 \mathrm{~mm}$.

of the different fibres produced here. The diameter of the fibres was $2 \pm 0.02 \mathrm{~mm}$ and they were manufactured using the highest piston speed during the capillary extrusion. There were clear signs of fibril agglomeration along the length of the composite fibres, seen as somewhat whitish areas in the micrographs; that is, the fibrils did not form a uniform network in the radial direction of the composite fibres throughout the fibre length and the fibrils were not unidirectional oriented, although a more preferred orientation of the fibrils along the fibre axis could probably be expected. In more detail, the fibrils formed an interconnecting structure in the axial direction of the fibres (as evident from the DMTAresults presented below), but the CNF distribution was not homogeneous in the radial direction.

The fibres containing T-CNF fibrils exhibited a yellowish tint compared to those with C-CNF. This effect has been observed by others in the case of TEMPO-oxidized cellulose, for example, as described by Takaichi et al. [43]. They reported that oven-drying of the cellulose promoted the formation of C6-aldehydes and C2/C3 ketones which contributed to the yellowing.

Scanning electron micrographs of typical fracture surfaces (see Figure 4) indicated that the fibrils were quite well distributed over the cross section at fibril contents of 10 and $30 \mathrm{wt}-\% \mathrm{C}-\mathrm{CNF}$ in some sections of the fibres. However, as already pointed to, this varied along the length of the fibres; in some sections there seemed to be less fibrils and then they were more concentrated towards certain parts of the cross section. No observable difference between the fracture surfaces was noted when C-CNF was exchanged for T-CNF. A closer examination of the fracture surfaces showed that there were very few signs of fibril pull-out from the matrix, which indicates a good adhesion between the fibrils and the matrix; compare [40]. This was the case even when the nongrafted fibrils were used as the reinforcing elements. Another interesting observation was that the (outer) fibre surface became smoother with increasing fibril content; see Figure 4 . This was also evident when touching (feeling) the fibres. The unfilled PEG-fibre exhibited the highest surface roughness. There could be different reasons for this behaviour. For example, the addition of the fibrils could stabilize the flow through the capillary in itself. It may also be that the surface irregularities appear as a result of the die-swell when the melt exits the capillary; compare [44]. Adding fibrils to the melt can reduce the die-swell (cf. [44]) and then reduce the surface roughness.

\subsection{Dynamic-Mechanical Thermal Analysis of the Composite} Fibres. As described above, two different kinds of measurements were performed, both using the same equipment. The dynamic strain sweep was performed at room temperature and ambient pressure. The reference sample, that is, the sample containing only PEG matrix material, exhibited an almost constant storage modulus $E^{\prime}$ in the linear viscoelastic region. The value of the storage modulus in this region was about $1 \mathrm{GPa}$ (more precise $0.84 \mathrm{GPa}$ with a standard deviation of $0.07 \mathrm{GPa}$ ) and the onset of the nonlinear behaviour occurred at strains around 0.1 as shown in Figure 5. Strainsweep experiments were also performed with the composite fibres containing 10 and 30 wt-\% C-CNF. Figure 5 includes an example of such a measurement in case of a fibre with a nominal C-CNF-content of $30 \mathrm{wt}-\%$. The storage modulus in the linear viscoelastic region was here about $2 \mathrm{GPa}$ (more precise $1.8 \mathrm{GPa}$ with standard deviation of $0.36 \mathrm{GPa}$ ); that is, the addition of the CNF increased the modulus. It may be remarked that in view of the inhomogeneous aggregate structure visualised in Figure 3 the variation in storage modulus of the composite fibres was quite low, indicating that $\mathrm{CNF}$ reinforced the polymer in a rather coherent manner along the fibre length.

The critical strain for the onset of nonlinear behaviour (corresponding in a sense to a disruption of the structure of the material) in case of the composite fibre shown in Figure 5 was about 0.05 . Adding fibrils to the PEG matrix thus decreased the linear viscoelastic region for the material, which is not unusual when fillers are incorporated into polymeric materials [45].

The onset of the nonlinear region denotes the maximum strain that can be applied when performing the temperature sweeps, since such measurements should be performed at conditions where the viscoelastic parameters are independent of the applied strain. Figure 6 shows $E^{\prime}$ as a function of the temperature for the reference PEG matrix material. In this case the temperature region was cut short since the material melted around $60^{\circ} \mathrm{C}$ and thus no signal was obtained at temperatures exceeding $65^{\circ} \mathrm{C}$. The applied strain amplitude here was 0.02 which is within the linear viscoelastic range according to the strain sweep. At temperatures below the melting point, the modulus decreased, as expected, somewhat with increasing temperature.

Figure 7 shows the temperature dependence of the storage modulus and of the mechanical loss factor $\tan \delta$ at $1 \mathrm{~Hz}$ for the same type of composite fibre as in Figure 5. The strain amplitude was set to 0.01 , that is, well below the critical strain.

In contrast to the behaviour of the unfilled PEG matrix, the storage modulus of the composite fibre did not decrease to a very low value at the melting point of the polymer. Instead the modulus was reduced about a decade and attained a "pseudo-" plateau level around $100 \mathrm{MPa}$, which decreased rather slowly with increasing temperature. The 


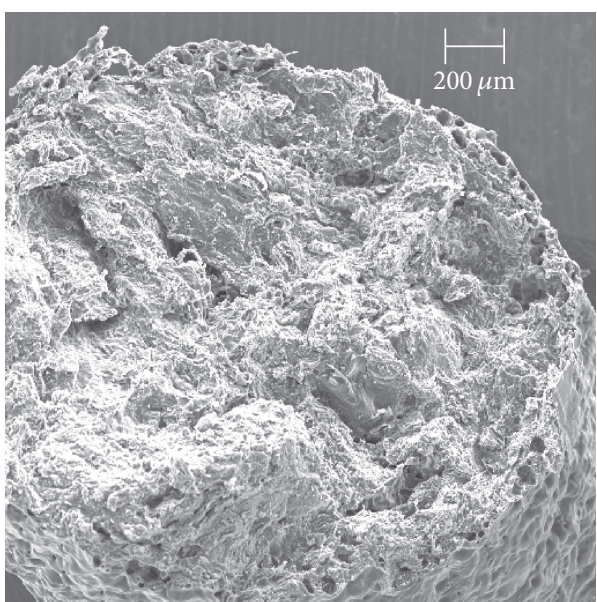

(a)

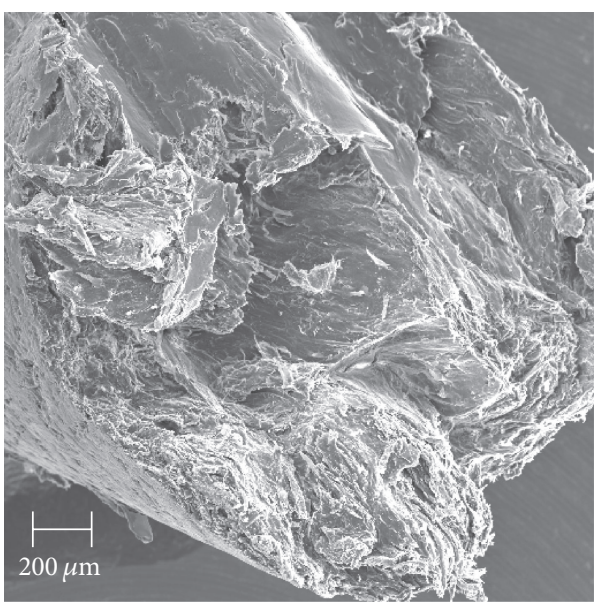

(b)

FIGURE 4: Scanning electron micrographs showing the fracture surface of a fibre containing $10 \mathrm{wt}$ - $\%$ C-CNF (a) and one containing $30 \mathrm{wt}$ - $\%$ C-CNF (b). The surface (outer) area can be seen in the bottom right corner (a) and bottom left corner (b) of the micrographs.

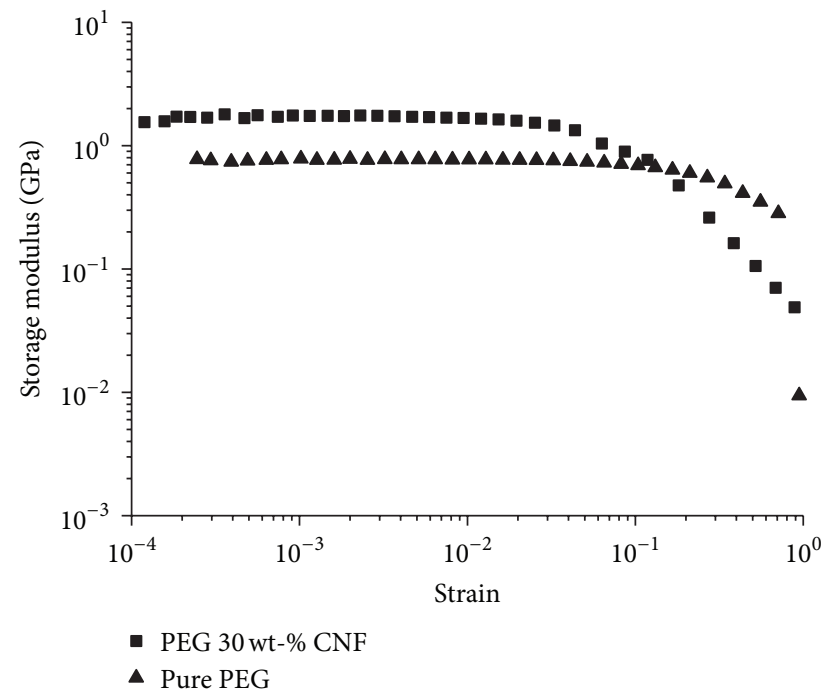

FIGURE 5: The storage modulus $E^{\prime}$ as a function of the applied strain amplitude at a frequency of $1 \mathrm{~Hz}$ for the PEG matrix (containing no $\mathrm{CNF}$ ) and for a composite fibre containing $30 \mathrm{wt}-\% \mathrm{C}-\mathrm{CNF}$.

plateau extended up to the highest temperature used here, $120^{\circ} \mathrm{C}$, without exhibiting any major changes. Since the PEG matrix melted already around $60^{\circ} \mathrm{C}$, it may be concluded that the quite high storage modulus is associated with a coherent and interconnected fibrillar network of CNF in the composite. This network was then formed when preparing the composite material and/or when the composite melt flowed through the capillary. The mechanical loss factor passed through a pronounced maximum in the melting region of the matrix polymer and then decreased rather slowly with increasing temperature when the melting point had been exceeded. It is not uncommon that $\tan \delta$ exhibits a high value when a polymer softens; compare, for example, [46].

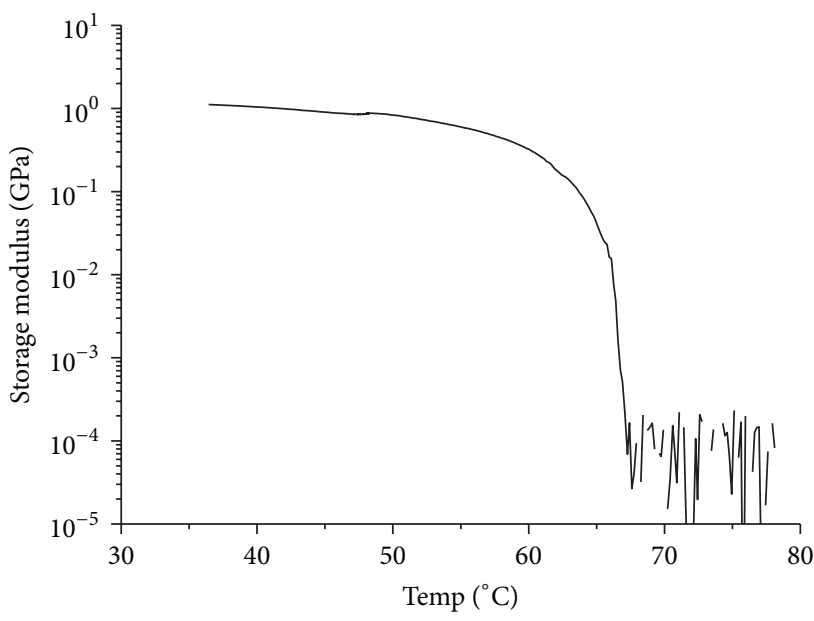

FIGURE 6: The storage modulus $E^{\prime}$ as a function of temperature for the PEG matrix (containing no CNF).

As shown in Figure 8, the composite fibre containing $10 \mathrm{wt}-\% \mathrm{C}-\mathrm{CNF}$ also exhibited a plateau at higher temperatures. The modulus associated with the plateau was however lower, approaching $10 \mathrm{MPa}$, than that of the fibre containing $30 \mathrm{wt}-\% \mathrm{C}-\mathrm{CNF}$, which is in line with the lower fibril content.

The composite fibres containing nominally $30 \mathrm{wt}-\%$ grafted and nongrafted T-CNF displayed a modulus-strain amplitude relation that was almost identical to that of the corresponding fibre containing the same amount of C-CNF; the extent of the linear viscoelastic region was not markedly affected. The strain amplitude used when performing the temperature sweep was 0.01 . Figure 9 shows the temperature dependence of the storage modulus for the composite fibres containing the grafted as well as the nongrafted T-CNF.

The temperature dependence of $E^{\prime}$ resembled to a large extent that of the composite fibre containing $30 \mathrm{wt}-\% \mathrm{C}-\mathrm{CNF}$. 


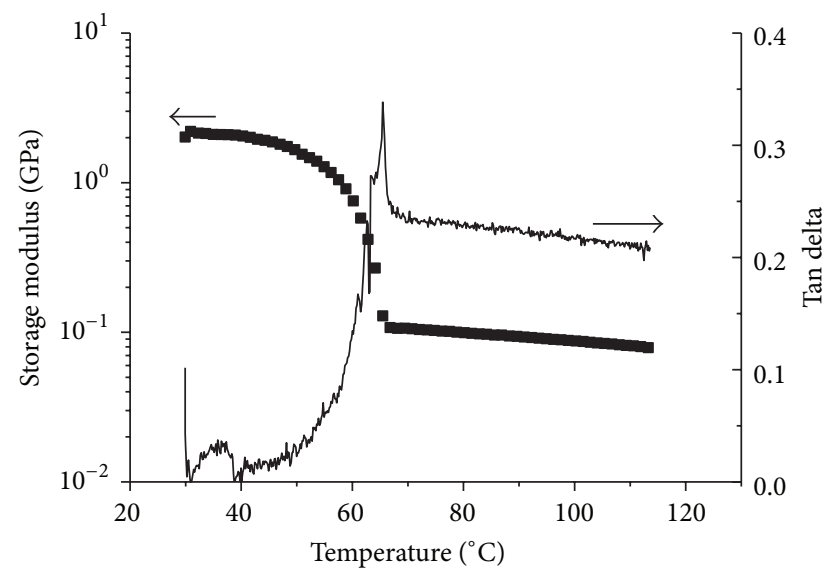

Figure 7: The storage modulus $E^{\prime}$ and the mechanical loss factor $\tan \delta$ at $1 \mathrm{~Hz}$ as functions of temperature for a fibre containing $30 \mathrm{wt}-$ $\% \mathrm{C}-\mathrm{CNF}$.

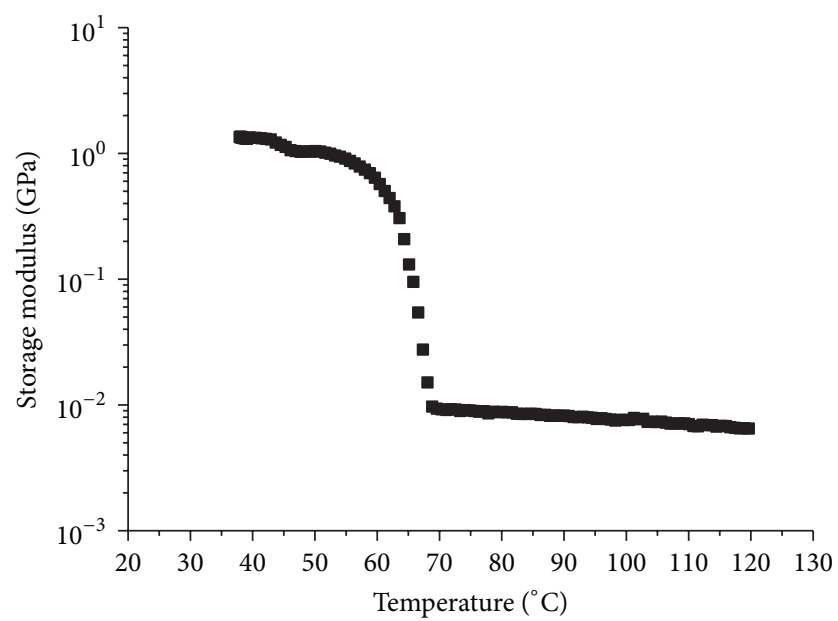

FIgURE 8: The storage modulus $E^{\prime}$ as a function of the temperature for a composite fibre containing $10 \mathrm{wt}-\mathrm{\%} \mathrm{C}-\mathrm{CNF}$.

Again a plateau in the storage modulus was noted at temperatures higher than the melting point of the matrix polymer for both the T-CNF-containing fibres. The corresponding value of the modulus was of the order of $100 \mathrm{MPa}$, being somewhat higher when the nongrafted T-CNF was used as the reinforcing phase. However, it should be remembered that the composite containing nominally $30 \mathrm{wt} \%$ grafted T-CNF actually contained only $20 \mathrm{wt}-\%$ cellulosic material indicating that the grafting promoted a stiffer (and probably stronger) fibrillar network in the composite fibres. This is quite plausible since the storage moduli of composite fibres containing nongrafted CNF apparently scaled with the CNFcontent; compare Figures 7 and 8.

\section{Discussion}

The addition of the CNF to the polymer matrix obviously had a strong effect on the modulus of the fibres; that is, with about 20 volume- $\% \mathrm{CNF}$, the modulus at room

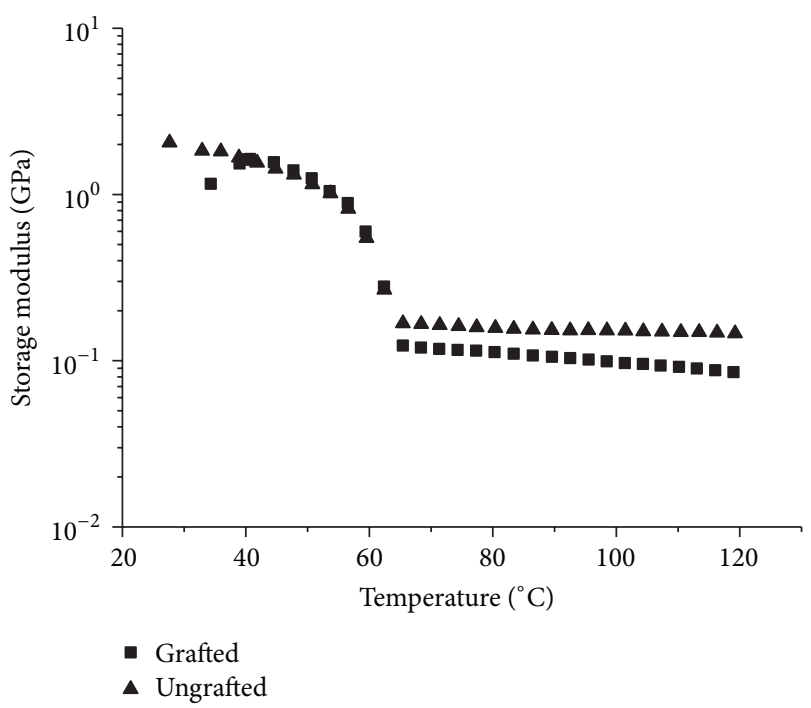

FIgURE 9: The storage modulus $E^{\prime}$ as a function of the temperature for composite fibres containing $30 \mathrm{wt}-\%$ grafted and nongrafted TCNF.

temperature was approximately doubled. Here, the weight fraction of the CNF (30 wt-\%) has been recalculated into a corresponding volume content, using a density of $1500 \mathrm{~kg} / \mathrm{m}^{3}$ for the CNF, giving about 20 volume-\%. The nanofibrils had also the expected effect on the melt viscosity of the composite melt; that is, it increased; compare, for example, [40], and the critical strain for onset of the structure deterioration decreased. With regard to the stiffness enhancement, no appreciable difference between $\mathrm{C}$-CNF and nongrafted T$\mathrm{CNF}$ was observed. The grafting appeared to improve the efficiency of the reinforcing elements, since about the same storage modulus of the composite fibres was noted as in the case of the nongrafted T-CNF although the cellulose content was about $30 \%$ lower. This may be interpreted as a result of an improved compatibility between the grafted PEG and the PEG matrix. Although this is plausible, the lack of extensive fibre pull-out in the fracture surfaces of all the composite fibres did indicate that the adhesion between the CNF and the matrix was in general quite good. The enhanced compatibility may then result in an improved dispersion of the CNF in the matrix which would promote the mechanical properties.

Both the mechanical performance and the micrographs show that the manner in which the composite fibres were produced counteracted, at least to some extent, the collapse of the fibril network when drying. Thus, the associated decrease in surface area of the fibrillary network was counteracted and the reinforcing effect of the fibrils was enhanced. This was also one of the aims of the preparation technique. Admittedly and as pointed to earlier, a perfect uniform distribution was not obtained and the micrographs, Figure 3, revealed that aggregates were clearly formed to some extent in the fibres. In a series of experiments, attempts were made to align the fibrillar network by orienting the composite fibres at elevated temperature and/or in a moistened state. These attempts were however not successful since either the polymer matrix was too brittle or its softening (melting) region was not 
sufficiently broad, both of which are likely to be associated with the rather low molecular weight of the PEG matrix (35000 g/mol). A higher degree of alignment would however be desirable from the mechanical performance point of view.

A striking feature of the temperature dependence of the modulus of the composite fibres is the second plateau at temperatures above the melting point of the polymer matrix; see Figures 7-9. The magnitude of the corresponding modulus scales with cellulose content (with exception of the composite containing the grafted T-CNF). An interpretation is that the CNF form a coherent network in the matrix (otherwise the modulus would decrease to zero). This network can result from the preparation of the composite material and/or be formed when the composite melt flows through the capillary. Visual examination of the fibres after the exposure to the high temperatures revealed that they consisted more or less only of cellulose fibrils in a collapsed state.

In this context, it is interesting to estimate how well the reinforcing ability of the CNF with regard to the stiffness is utilized in the composite fibres. The modulus of a composite can be modelled in several ways; compare, for example, [34], and the modelling involves a number of assumptions and estimations. The fibrils are assumed to be straight, with cylindrical cross section and without any defects, which clearly is not the case here, and it is assumed that the adhesion between the matrix and the reinforcement is sufficient. A rather simple model that can be used for the intended purpose is that of Cox-Krenchel [19, 34, 47]:

$$
E_{c}=\eta_{d} \eta_{l} v_{f} E_{f}+\left(1-v_{f}\right) E_{m}
$$

where $E_{c}$ is the elastic modulus of the composite fibres in the fibre direction, $v_{f}$ the volume fraction of CNF, $E_{f}$ the (effective) modulus of the fibrils in the axial direction, and $E_{m}$ the modulus of the matrix (here taken to be $0.9 \mathrm{GPa}$ from the measurements of the storage modulus). The factor $\eta_{d}$ accounts for the orientation of the fibrils; here it is assumed that the fibrils are randomly oriented in three dimensions (as roughly estimated from the optical micrographs) and in such a case $\eta_{d}=0.2$ [46] (which might be an underestimation). The correction for a finite fibril length given by $\eta_{l}$ stems from the shear-lag theory [34] and is given by

$$
\eta_{l}=1-\frac{\tanh (m a)}{m a} .
$$

Here $a$ is the aspect ratio of the fibrils, that is, the ratio between the fibril length $(l)$ and the diameter $(d)$. From the data on fibril dimensions reported by Wågberg et al. [7], $a=$ 100 appears to be a reasonable value; the analysis performed here is actually not very sensitive to values of $a$ between 50 and 200. The factor $m$ is obtained from

$$
m=\sqrt{\frac{2 G_{m}}{E_{f} \ln (2 R / d)}},
$$

where $G_{m}$ is the shear modulus of the matrix and $2 R$ is the distance between the fibrils. The shear modulus can be estimated from the storage modulus of PEG and assuming that the fibrils are arranged in square array, the ratio $R / d$ can be related to the volume fraction of fibrils $v_{f}$.

With these assumptions and with a volume fraction of fibrils of 0.2 , an effective fibril modulus $E_{f}$ of about $34 \mathrm{GPa}$ was obtained using the values of the storage modulus at room temperature of the composite fibres containing C-CNF. At a volume content of 0.16 , corresponding to composite containing grafted T-CNF (only accounting for the cellulosic material), the effective modulus was $44 \mathrm{GPa}$ and a further decrease of the volume content to 0.08 , corresponding to the fibre containing 10 wt- $\% \mathrm{C}$-CNF, increased the evaluated $E_{f}$-value to more than $60 \mathrm{GPa}$. The calculated values of the effective modulus may be somewhat overestimated (mainly due to the assumption regarding the fibril distribution), but the trend is clear. With decreasing fibril content, the effective fibril modulus increases. Similar results were reported in [23, 38], using the Halpin-Tsai model, where CNF were incorporated into polyester and epoxy matrices. It was suggested that agglomeration at higher fibril contents could lead to a less efficient load transfer between the fibrils and the matrix (and a lower available surface area of the fibrils) resulting in dependence of the effective modulus on the fibril content, which seems quite plausible. The calculated values of the fibril modulus are high, but clearly below the possible limits $[19,33]$ which gives room for improvements with regard to the mechanical performance of the polymer-based composites in terms of a more homogeneous fibril distribution and, in the present case, an enhanced alignment of the CNF in the fibre direction.

\section{Conclusions}

The addition of the CNF to the polymer matrix clearly enhanced the stiffness of the composite fibres and decreased the critical strain for onset of the structure deterioration. The grafting of PEG on the T-CNF improved the efficiency of the reinforcing elements, since about the same storage modulus (stiffness) of the composite fibres was noted as in the case of the nongrafted T-CNF although the cellulose content was about $30 \%$ lower. This can be interpreted in terms of an improved compatibility between the grafted PEG and the PEG matrix, possibly associated with an enhanced dispersion of the CNF in the matrix.

The DMTA-measurements clearly revealed a second plateau in the modulus-temperature curves for the composite fibres at temperatures exceeding the melting point of the PEG matrix. It is suggested that this plateau can be associated with fibrillary network that is coherent and interconnected in the axial direction of the composite fibres, although the distribution of the CNF was not homogeneous in the radial direction. The analysis of the experimental stiffness results pointed to the fact that agglomeration at higher fibril contents could lead to less efficient load transfer between the fibrils and the matrix (and a lower available surface area of the fibrils) and thus a lower effective reinforcement provided by the CNF.

\section{Competing Interests}

The authors declare that they have no competing interests. 


\section{Acknowledgments}

The authors acknowledge Wallenberg Wood Science Center and Chalmers University of Technology for the financial support.

\section{References}

[1] A. J. Ragauskas, C. K. Williams, B. H. Davison et al., "The path forward for biofuels and biomaterials," Science, vol. 311, no. 5760, pp. 484-489, 2006.

[2] K. Oksman, Y. Aitomäki, A. P. Mathew et al., "Review of the recent developments in cellulose nanocomposite processing," Composites A: Applied Science and Manufacturing, vol. 83, pp. 2-18, 2016.

[3] M. Thunwall, V. Kuthanová, A. Boldizar, and M. Rigdahl, "Film blowing of thermoplastic starch," Carbohydrate Polymers, vol. 71, no. 4, pp. 583-590, 2008.

[4] S. J. Eichhorn, C. A. Baillie, N. Zafeiropoulos et al., "Current international research into cellulosic fibres and composites," Journal of Materials Science, vol. 36, no. 9, pp. 2107-2131, 2001.

[5] M. Pääkko, M. Ankerfors, H. Kosonen et al., "Enzymatic hydrolysis combined with mechanical shearing and high-pressure homogenization for nanoscale cellulose fibrils and strong gels," Biomacromolecules, vol. 8, no. 6, pp. 1934-1941, 2007.

[6] A. Isogai, T. Saito, and H. Fukuzumi, "TEMPO-oxidized cellulose nanofibers," Nanoscale, vol. 3, no. 1, pp. 71-85, 2011.

[7] L. Wågberg, G. Decher, M. Norgren, T. Lindström, M. Ankerfors, and K. Axnäs, "The build-up of polyelectrolyte multilayers of microfibrillated cellulose and cationic polyelectrolytes," Langmuir, vol. 24, no. 3, pp. 784-795, 2008.

[8] $\varnothing$. Eriksen, K. Syverud, and Ø. Gregersen, “The use of microfibrillated cellulose produced from kraft pulp as strength enhancer in TMP paper," Nordic Pulp and Paper Research Journal, vol. 23, no. 3, pp. 299-304, 2008.

[9] T. Taipale, M. Österberg, A. Nykänen, J. Ruokolainen, and J. Laine, "Effect of microfibrillated cellulose and fines on the drainage of kraft pulp suspension and paper strength," Cellulose, vol. 17, no. 5, pp. 1005-1020, 2010.

[10] M. Henriksson, L. A. Berglund, P. Isaksson, T. Lindström, and T. Nishino, "Cellulose nanopaper structures of high toughness," Biomacromolecules, vol. 9, no. 6, pp. 1579-1585, 2008.

[11] M. Ankerfors, T. Lindström, and D. Söderberg, "The use of microfibrillated cellulose in fine paper manufacturing-results from a pilot scale papermaking trial," Nordic Pulp and Paper Research Journal, vol. 29, no. 3, pp. 476-483, 2014.

[12] C. Aulin, M. Gällstedt, and T. Lindström, "Oxygen and oil barrier properties of microfibrillated cellulose films and coatings," Cellulose, vol. 17, no. 3, pp. 559-574, 2010.

[13] H. Fukuzumi, T. Saito, T. Iwata, Y. Kumamoto, and A. Isogai, "Transparent and high gas barrier films of cellulose nanofibers prepared by TEMPO-mediated oxidation," Biomacromolecules, vol. 10, no. 1, pp. 162-165, 2009.

[14] I. Siró, D. Plackett, M. Hedenqvist, M. Ankerfors, and T. Lindström, "Highly transparent films from carboxymethylated microfibrillated cellulose: the effect of multiple homogenization steps on key properties," Journal of Applied Polymer Science, vol. 119, no. 5, pp. 2652-2660, 2011.

[15] D. Klemm, F. Kramer, S. Moritz et al., "Nanocelluloses: a new family of nature-based materials," Angewandte Chemie-International Edition, vol. 50, no. 24, pp. 5438-5466, 2011.
[16] C. Chang and L. Zhang, "Cellulose-based hydrogels: present status and application prospects," Carbohydrate Polymers, vol. 84, no. 1, pp. 40-53, 2011.

[17] H. Jin, M. Kettunen, A. Laiho et al., "Superhydrophobic and superoleophobic nanocellulose aerogel membranes as bioinspired cargo carriers on water and oil," Langmuir, vol. 27, no. 5, pp. 1930-1934, 2011.

[18] H. Sehaqui, M. Salajková, Q. Zhou, and L. A. Berglund, "Mechanical performance tailoring of tough ultra-high porosity foams prepared from cellulose I nanofiber suspensions," Soft Matter, vol. 6, no. 8, pp. 1824-1832, 2010.

[19] K.-Y. Lee, Y. Aitomäki, L. A. Berglund, K. Oksman, and A. Bismarck, "On the use of nanocellulose as reinforcement in polymer matrix composites," Composites Science and Technology, vol. 105, pp. 15-27, 2014.

[20] K. Abe, F. Nakatsubo, and H. Yano, "High-strength nanocomposite based on fibrillated chemi-thermomechanical pulp," Composites Science and Technology, vol. 69, no. 14, pp. 24342437, 2009.

[21] I. Siró and D. Plackett, "Microfibrillated cellulose and new nanocomposite materials: a review," Cellulose, vol. 17, no. 3, pp. 459-494, 2010.

[22] M. Jonoobi, A. P. Mathew, M. M. Abdi, M. D. Makinejad, and K. Oksman, "A comparison of modified and unmodified cellulose nanofiber reinforced polylactic acid (PLA) prepared by twin screw extrusion," Journal of Polymers and the Environment, vol. 20, no. 4, pp. 991-997, 2012.

[23] F. Ansari, M. Skrifvars, and L. Berglund, "Nanostructured biocomposites based on unsaturated polyester resin and a cellulose nanofiber network," Composites Science and Technology, vol. 117, pp. 298-306, 2015.

[24] A. Boldizar, C. Klason, J. Kubát, P. Näslund, and P. Sáha, "Prehydrolyzed cellulose as reinforcing filler for thermoplastics," International Journal of Polymeric Materials, vol. 11, no. 4, pp. 229-262, 1987.

[25] J. K. Pandey, A. N. Nakagaito, and H. Takagi, "Fabrication and applications of cellulose nanoparticle-based polymer composites," Polymer Engineering and Science, vol. 53, no. 1, pp. 1-8, 2013.

[26] T. Moberg and M. Rigdahl, "On the viscoelastic properties of microfibrillated cellulose (MFC) suspensions," Transactions of the Nordic Rheology Society, vol. 20, pp. 123-130, 2012.

[27] A. Naderi, T. Lindström, and J. Sundström, "Carboxymethylated nanofibrillated cellulose: rheological studies," Cellulose, vol. 21, no. 3, pp. 1561-1571, 2014.

[28] L. Jowkarderis and T. G. M. van de Ven, "Rheology of semidilute suspensions of carboxylated cellulose nanofibrils," Carbohydrate Polymers, vol. 123, pp. 416-423, 2015.

[29] A.-H. Vesterinen, P. Myllytie, J. Laine, and J. Seppälä, “The effect of water-soluble polymers on rheology of microfibrillar cellulose suspension and dynamic mechanical properties of paper sheet," Journal of Applied Polymer Science, vol. 116, no. 5, pp. 2990-2997, 2010.

[30] A. Karppinen, A.-H. Vesterinen, T. Saarinen, P. Pietikäinen, and J. Seppälä, "Effect of cationic polymethacrylates on the rheology and flocculation of microfibrillated cellulose," Cellulose, vol. 18, no. 6, pp. 1381-1390, 2011.

[31] M.-P. Lowys, J. Desbrières, and M. Rinaudo, "Rheological characterization of cellulosic microfibril suspensions. Role of polymeric additives," Food Hydrocolloids, vol. 15, no. 1, pp. 25-32, 2001. 
[32] T. Moberg, M. Rigdahl, M. Stading, and E. Levenstam Bragd, "Extensional viscosity of microfibrillated cellulose suspensions," Carbohydrate Polymers, vol. 102, no. 1, pp. 409-412, 2014.

[33] I. Sakurada, Y. Nukushina, and T. Ito, "Experimental determination of the elastic modulus of crystalline regions in oriented polymers," Journal of Polymer Science, vol. 57, no. 165, pp. 651660, 1962.

[34] N. G. McCrum, C. P. Buckley, and C. B. Bucknall, Principles of Polymer Engineering, Oxford Science Publications, Oxford, UK, 1997.

[35] S. Iwamoto, A. Isogai, and T. Iwata, "Structure and mechanical properties of wet-spun fibers made from natural cellulose nanofibers," Biomacromolecules, vol. 12, no. 3, pp. 831-836, 2011.

[36] J. G. Torres-Rendon, F. H. Schacher, S. Ifuku, and A. Walther, "Mechanical performance of macrofibers of cellulose and chitin nanofibrils aligned by wet-stretching: a critical comparison," Biomacromolecules, vol. 15, no. 7, pp. 2709-2717, 2014.

[37] K. M. O. Håkansson, A. B. Fall, F. Lundell et al., "Hydrodynamic alignment and assembly of nanofibrils resulting in strong cellulose filaments," Nature Communications, vol. 5, article 4018, 2014.

[38] F. Ansari, S. Galland, M. Johansson, C. J. G. Plummer, and L. A. Berglund, "Cellulose nanofiber network for moisture stable, strong and ductile biocomposites and increased epoxy curing rate," Composites Part A: Applied Science and Manufacturing, vol. 63, pp. 35-44, 2014.

[39] H. Tang, N. Butchosa, and Q. Zhou, "A transparent, hazy, and strong macroscopic ribbon of oriented cellulose nanofibrils bearing poly(ethylene glycol)," Advanced Materials, vol. 27, no. 12, pp. 2070-2076, 2015.

[40] M. Thunwall, A. Boldizar, M. Rigdahl et al., "Processing and properties of mineral-interfaced cellulose fibre composites," Journal of Applied Polymer Science, vol. 107, no. 2, pp. 918-929, 2008.

[41] H. A. Barnes, J. F. Hutton, and K. Walters, An Introduction to Rheology, chapter 7, Elsevier Science, Amsterdam, The Netherlands, 1989.

[42] P. Gatenholm, H. Bertilsson, and A. Mathiasson, "Effect of chemical composition of interphase on dispersion of cellulose fibers in polymers. I. PVC-coated cellulose in polystyrene," Journal of Applied Polymer Science, vol. 49, no. 2, pp. 197-208, 1993.

[43] S. Takaichi, T. Saito, R. Tanaka, and A. Isogai, "Improvement of nanodispersibility of oven-dried TEMPO-oxidized celluloses in water," Cellulose, vol. 21, no. 6, pp. 4093-4103, 2014.

[44] J. M. Dealey and K. F. Wissbrun, Melt Rheology and Its Role in Plastics Processing, Van Nostrand Reihold, New York, NY, USA, 1990.

[45] H. Bertilsson, On the transition to marked nonlinear viscoelasticity in solid polymer [Ph.D. thesis], Royal Institute of Technology, Stockholm, Sweden, 1977.

[46] H. A. Barnes, A Handbook of Elementary Rheology, vol. 13, chapter 13, University of Wales, Cambrian Printers, Aberystwyth, UK, 2000.

[47] F. L. Matthews and R. D. Rawlings, Composite Materials: Engineering and Science, Chapman \& Hall, London, UK, 1994. 

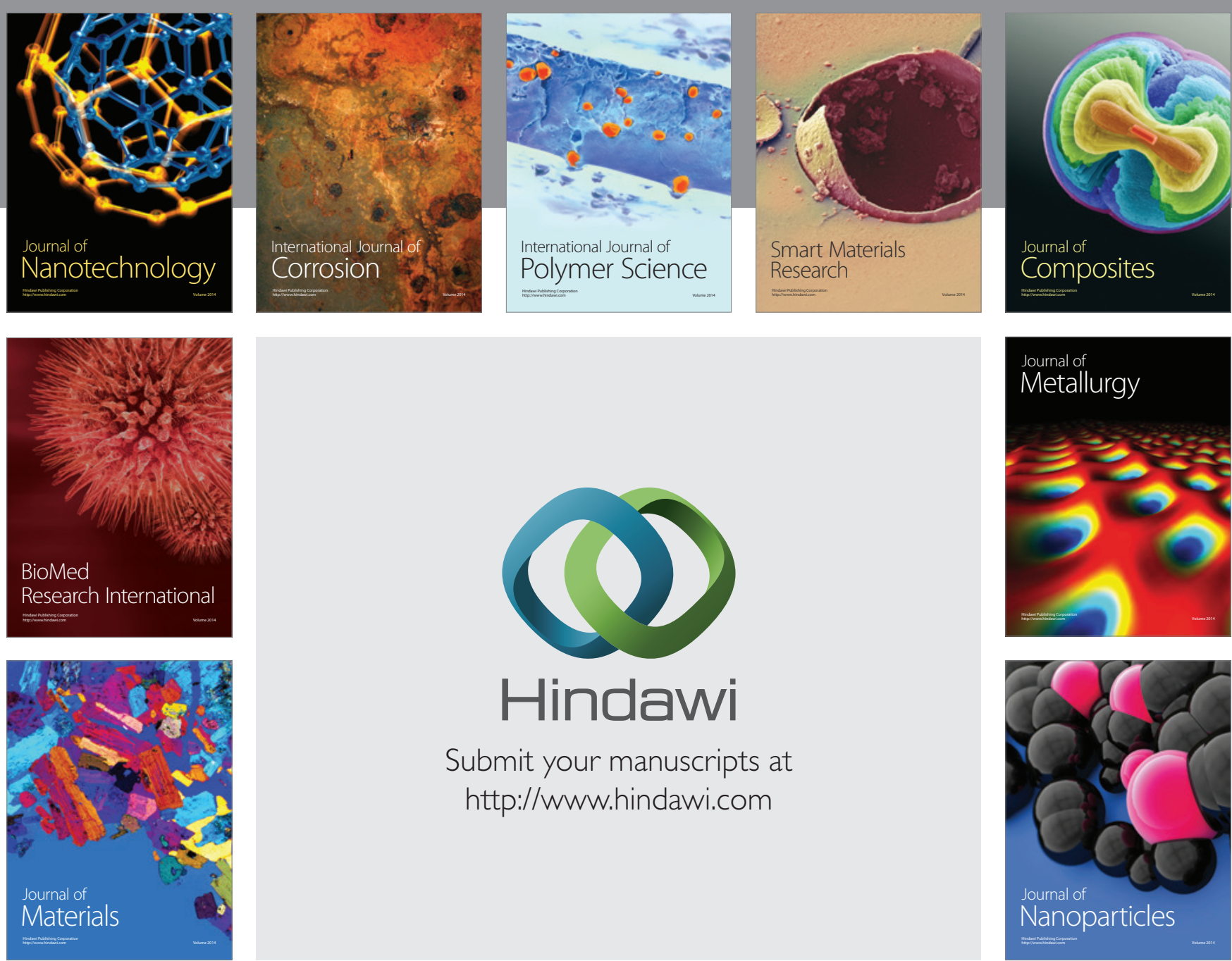

\section{Hindawi}

Submit your manuscripts at

http://www.hindawi.com

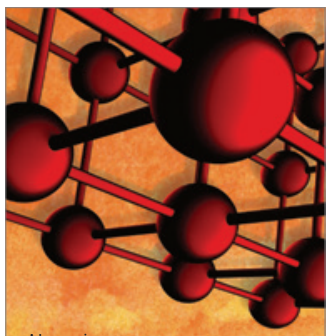

Materials Science and Engineering
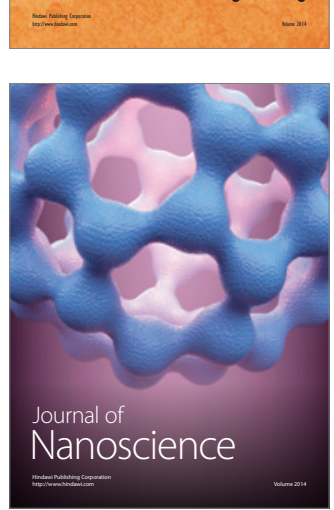
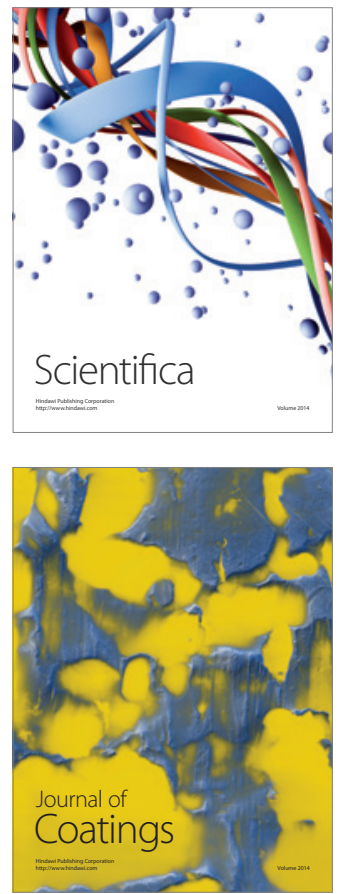
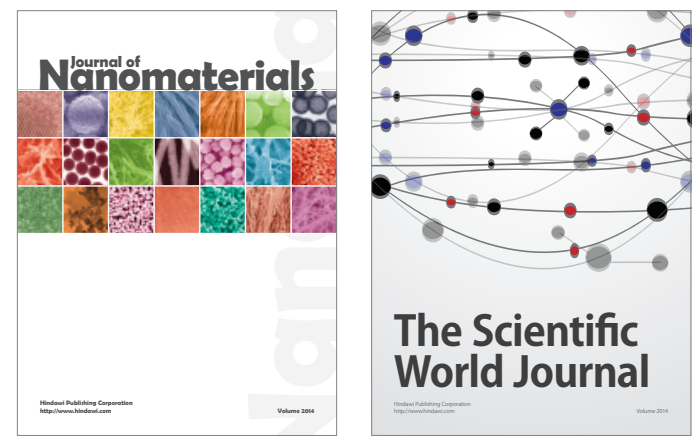

The Scientific World Journal
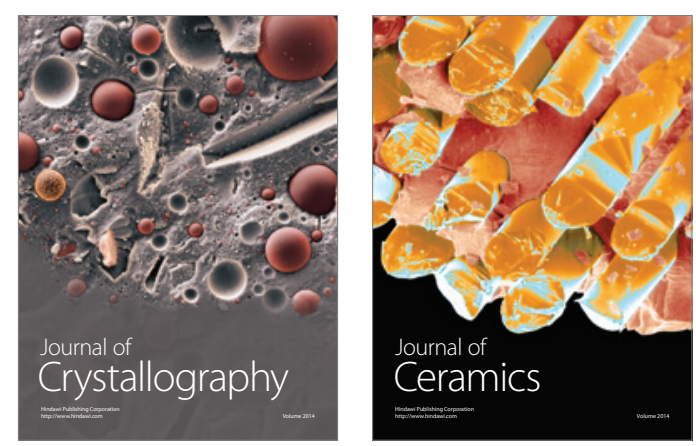
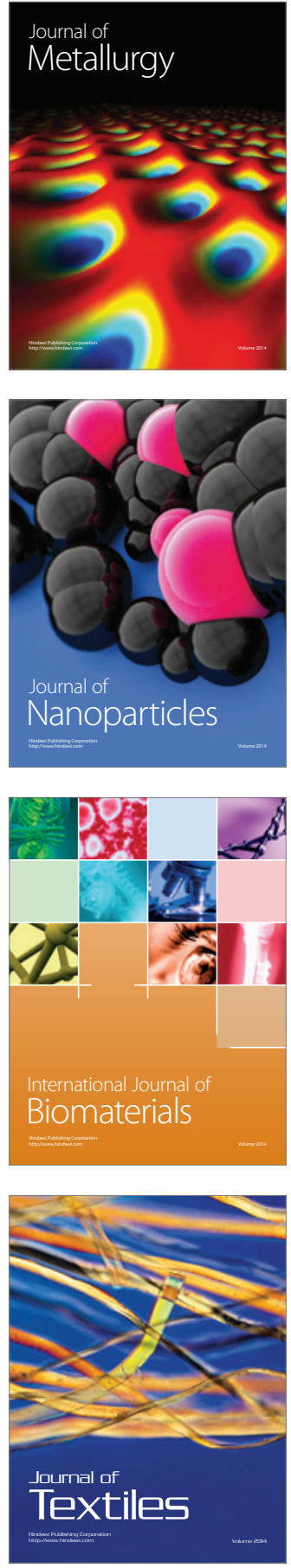